\title{
Focus point: classical and quantum information geometry
}

\author{
F. M. Ciagliaa ${ }^{1, \mathrm{a}} \mathbb{D}_{\mathbb{D}}$, S. Mancini $^{2, \mathrm{~b}}{ }_{\mathbb{D}}$, M. Ha Quang $^{3, \mathrm{c}}{ }_{\mathbb{D}}$ \\ ${ }_{1}^{1}$ Max Planck Institute for Mathematics in the Sciences, Leipzig, Germany \\ 2 University of Camerino, Camerino, Italy \\ ${ }^{3}$ RIKEN Center for Advanced Intelligence Project, Tokyo, Japan
}

(C) The Author(s) 2021

The interplay between Differential Geometry, Mathematical Statistics, Probability Theory and Quantum Mechanics resulting in the so-called field of Classical and Quantum Information Geometry represents one of the most effective examples of cross-fertilizations of modern Science. Roughly speaking, Information Geometry deals with the understanding of the differential geometric properties of suitable manifolds of Classical probability distributions and Quantum states, and with the concrete possible applications that such a theoretical understanding necessarily triggers.

Arguably, the genesis of Information Geometry could be traced back to the observation made by Fisher and then developed by Mahalanobis and Rao that, given suitable parametrized families of probability distributions, there is a "natural" choice of a Riemannian metric tensor on the manifold of parameters. This Riemannian metric tensor, known as the Fisher-Rao metric tensor, turned out to be particularly meaningful in Statistics, for instance in the problem of parameter estimation where it enters in the so-called Cramer-Rao bound providing a lower bound on the variance of unbiased estimators. The intrinsic significance of the Fisher-Rao metric tensor was later clarified by Cencov's work where it is showed that the Fisher-Rao metric tensor is the unique (up to a multiplicative constant) metric tensor on the interior of the simplex of probability distributions on a finite outcome space satisfying a categorical invariance property. Starting from these premises, the investigation of the geometrical properties of suitable manifolds of probability distributions proceeded in different directions. For instance, it was realized by Effron that the role of the embedding curvature of the parameter space is connected with an affine connection which is not the Levi-Civita connection of the Fisher-Rao metric tensor. This idea has then been developed into the definition of the so-called dualistic structure on the parameter manifold, namely, a couple of affine connections which are dual, in a suitable sense, with respect to the Fisher-Rao metric tensor. Also, the infinitesimal geometrical properties encoded in the Fisher-Rao metric tensor and the dualistic structure where found to be intimately connected with various notion of relative entropies between probability distributions, e.g., the Kullback-Leibler relative entropy, Tsallis relative entropy, Csizar relative entropy. Interestingly, it happens that, performing a suitable expansion of

Focus Point on Classical and Quantum Information Geometry.

\footnotetext{
a e-mail: ciaglia@mis.mpg.de (corresponding author)

b e-mail: stefano.mancini@unicam.it

c e-mail: minh.haquang@ riken.jp
} 
these relative entropies, the second-order contribution always coincides with the Fisher-Rao metric tensor, while the third-order contribution gives rise to a symmetric, 3-covariant tensor field inducing different dualistic structures depending on the relative entropy considered. In a certain sense, relative entropies determine the geometry of the parameter manifold.

Of course, all the developments made in the Classical realm of Statistics called for a similar investigation in the Quantum realm where probability distributions are replaced by quantum states. For instance, Helstrom analysed the problem of parameter estimation of quantum states and discovered a Riemannian metric tensor which is connected with a quantum analogue of the Cramer-Rao bound, and thus plays a role analogue to the Fisher-Rao metric tensor. The distance induced by this Riemannian metric tensor is given by the so-called Bures distance which is connected with the notion of transition probability between quantum states introduced by Uhlmann and Cantoni. However, it was discovered by Petz that, for finitedimensional quantum system, Cencov's uniqueness result is violated, and the Riemannian metric discovered by Helstrom is thus not the only meaningful one. For instance, unlike what happens in the Classical case, performing a suitable expansion of the quantum relative entropies one obtains a second-order contributions that is different for different quantum relative entropies, e.g., the von Neumann-Umegaki relative entropy, which is a generalization of the Kullback-Leibler relative entropy, leads to the so-called Bogoliubov-Kubo-Mori metric tensor, while the Bures distance leads to the Riemannian metric discovered by Helstrom. The investigation of the so-called monotone quantum metrics classified by Petz is an active field of investigation in Quantum Information Geometry, and its infinite-dimensional extension is one of the major open problems of this field.

Regarding the applicative side of Information Geometry, it is almost impossible to give an exhaustive list of the specific topics covered by Information Geometry and its methods, but it is worth mentioning that it has impact in fields like (Classical and Quantum) Information theory, (Classical and Quantum) Parametric Estimation Theory, Machine Learning, Deep Learning, Neural Networks, Optimization Theory, Artificial Intelligence, Signal Theory, Quantum Computation, Quantum Metrology, Neuroscience, and Complex Systems.

This Focus Point consists of 7 contributions ranging in different subfields of Classical and Quantum Information Geometry, and its aim is to present some of the more recent developments in these frameworks to readers who have different scientific backgrounds and work in different fields of application of Information Geometry. With this selection, we hope to contribute a little more to the transversal spreading of the beautiful subject of Information Geometry.

The first contribution [1] deals with the study of the dually flat structure induced by the monotone metric tensors on the space of faithful states of a two-level quantum system (i.e., a q-bit).

In the second contribution [2], any operator graph is associated with a number of quantum channels and the possibility of transmitting quantum information though a quantum channel with zero error is determined by the geometrical properties of the corresponding graph.

The third contribution [3] provides a unifying variational calculus derivation of Groverian geodesics for both quantum state vectors and quantum probability amplitudes.

In the fourth contribution [4], the possibility of relaxing the monotonicity property characteristic of the Riemannian metrics in Petz's classification is investigated .

In the fifth contribution [5], a parameter-free description of the manifold of faithful quantum states of a finite-dimensional quantum system is given.

The sixth contribution [6] develops quantum-computational schemes for supervised and unsupervised classification tasks by employing the quantum information-geometric tools. 
In the seventh contribution [7], the authors develop a functorial language of probabilistic morphisms and apply this framework to several basic problems in Bayesian nonparametrics.

As Guest Editors we wish to express our gratitude to the authors of the articles and the reviewers, for their efforts in ensuring the high quality of the work and its presentation.

Funding Open Access funding enabled and organized by Projekt DEAL.

Open Access This article is licensed under a Creative Commons Attribution 4.0 International License, which permits use, sharing, adaptation, distribution and reproduction in any medium or format, as long as you give appropriate credit to the original author(s) and the source, provide a link to the Creative Commons licence, and indicate if changes were made. The images or other third party material in this article are included in the article's Creative Commons licence, unless indicated otherwise in a credit line to the material. If material is not included in the article's Creative Commons licence and your intended use is not permitted by statutory regulation or exceeds the permitted use, you will need to obtain permission directly from the copyright holder. To view a copy of this licence, visit http://creativecommons.org/licenses/by/4.0/.

\section{References}

1. A. Fujiwara, Dually flat structures induced from monotone metrics on a two-level quantum state space. Eur. Phys. J. Plus 135(10), 860 (2020). https://doi.org/10.1140/epjp/s13360-020-00877-9

2. G.G. Amosov, On inner geometry of noncommutative operator graphs. Eur. Phys. J. Plus 135(10), 865 (2020). https://doi.org/10.1140/epjp/s13360-020-00871-1

3. C. Cafaro, D. Felice, P.M. Alsing, Quantum Groverian geodesic paths with gravitational and thermal analogies. Eur. Phys. J. Plus 135(11), 900 (2020). https://doi.org/10.1140/epjp/s13360-020-00914-7

4. J. Suzuki, Non-monotone metric on the quantum parametric model. Eur. Phys. J. Plus 136(1), 90 (2021). https://doi.org/10.1140/epjp/s13360-021-01101-y

5. J. Naudts, Parameter-free description of the manifold of non-degenerate density matrices. Eur. Phys. J. Plus 136(1), 93 (2021). https://doi.org/10.1140/epjp/s13360-020-01038-8

6. F. Shahi, A.T. Rezakhani, Fidelity-based supervised and unsupervised learning for binary classification of quantum states. Eur. Phys. J. Plus 136(3), 280 (2021). https://doi.org/10.1140/epjp/s13360-021-01232-2

7. J. Jost, H.V. Le, T.D. Tran, Probabilistic morphisms and Bayesian nonparametrics. Eur. Phys. J. Plus 136(1), 441 (2021). https://doi.org/10.1140/epjp/s13360-021-01427-7 